\title{
E-Pharmacophore Model Assisted Discovery of Novel Antagonists of nNOS
}

\author{
Nalamolu Ravina Madhulitha, Natarajan Pradeep, Swargam Sandeep, Kanipakam Hema, Pasala Chiranjeevi, Katari Sudheer Kumar and \\ Amineni Uma-Maheswari
}

Bioinformatics Centre, Department of Bioinformatics, SVIMS University, Tirupati-517507, Andhra Pradesh, India

\begin{abstract}
The nitric oxide (NO) synthesized by neuronal nitric oxide synthase (nNOS) acts as a neurotransmitter and plays a crucial role in a series of neurobiological functions. In diseased condition, activated nNOS induces nitrosylation as well as phosphorylation of tau protein and glycogen synthase kinase 3 beta (GSK-3 $\beta$ ) respectively. Hyper phosphorylation of tau accelerates tau oligomerization resulting in formation of neurofibrillary tangles (NFT), ensuring the neuronal cell death in hippocampus region; a hallmark of Alzheimer's disease (AD). Thus, designing inhibitor towards nNOS may reduce the neuronal loss caused by nNOS. Hence nNOS has been one of the revitalizing targets for $A D$. In the present work, one energetically optimized structure-based pharmacophore (e-pharmacophore) was generated using nNOS co-crystal structure $(4 \mathrm{D} 1 \mathrm{~N})$ to map important pharmacophoric features of nNOS. Shape based similarity screening performed using e-pharmacophore against in-house library of more than one million compounds resulted 2701 library of compounds. Rigid receptor docking (RRD) was applied and followed by molecular mechanics and generalized Born and surface area (MM-GBSA) calculation which results 22 nNOS ligands. To define the leads, dock complexes were subjected to quantum-polarized ligand docking (QPLD) followed by free energy calculations revealed 3 leads. On comparison with 1 existing inhibitor,it concealed three best leads with lower binding energy and better binding affinity. The best lead was subjected to induced fit docking (IFD) with MM-GBSA calculation and further molecular dynamics (MD) simulations for $50 \mathrm{~ns}$ in solvated model system. Potential energy, root mean square deviation (RMSD) and root mean square fluctuations (RMSF) results disclosed constancy of lead 1 interactions throughout $50 \mathrm{~ns}$ MD simulations run. Thus proposed three leads are having favorable absorption distribution metabolism excretion toxicity (ADME/T) properties and provide a scaffold for designing nNOS antagonists.
\end{abstract}

Keywords: nNOS; Alzheimer's disease; E-pharmacophore; Docking; MD simulations

\section{Introduction}

Alzheimer's disease (AD) is the most common form of dementia characterized clinically with progressive cognitive decline and neuronal loss [1]. Pathologically, AD affected brain shows accumulation of $\beta$-amyloid $(A \beta)$ plaques and neurofibrillary tangles formed by the hyper phosphorylation of tau protein [2]. Recent studies indicate that mitochondrial dysfunction, a pathological feature that can be detected early in AD [3]. Nitric oxide synthases (NOS) are a family of enzymes catalyzing the production of nitric oxide (NO), which functions as neuronal signaling molecule [4]. Altered expression of nNOS by the A $\beta$ stimulus results in the formation of peroxy nitrite and reactive oxygen species [5]. These peroxy-nitrite, nitrosylates the cytoplasmic protein tau and phosphorylates GSK-3 $\beta$ [5]

Nitrosylation of tau protein results in structural and conformational change of tau protein ensuring the destabilization of microtubule [6]. This leads to dissociation of tau protein from microtubule and oligomerization of tau occurs to form neuro-fibrillary tangles (NFT's). These tangles forms a physical road blocks within the neuronal cells and interrupts the neurotransmitter signaling. Destabilized microtubule leads to the loss of neuronal cell structure ensuing the vacuole in hippocampus region of brain another hallmark of AD [7]. nNOS mediated phosphorylation of GSK- $3 \beta$ gets activated and further phosphorylates tau protein which ensues the formation of NTF's [7].

The reactive oxygen species formed due to altered expression of nNOS damages the mitochondrial membrane and is associated with impairment of energy homeostasis deficit in the function of complexes of the respiratory chains reduced ATP synthesis as well as altered mitochondrial structure. This ensues the production of cytochrome $\mathrm{C}$ which activates the apoptotic protein $\mathrm{BCl}-2$, resulting in the neuronal cell death by stimulating neuronal apoptosis [8]. Following such NFT's aggregation, disintegration of microtubules, collapsing the neuron's transport system with consequent altered communication between neurons, eventually ending in neuronal cell death [9].

Reduced hyper phosphorylation of tau can be achieved with the inhibition of nNOS, which in turn reduces formation of neuro-fibrillary tangles in the hippocampus region of brain [10]. In silico approaches such as e-pharmacophore modelling followed by multiple docking would be helpful in defining nNOS inhibitors. Three e-pharmacophore models defined increases the diversity of the compound and used for pharmacophore based similarity search against more than 21 million compounds. The ability of defined e-pharmacophores to retrieve actives was evaluated or validated using receiver operative characteristic curve (ROC). Multiple docking strategies (rigid receptor docking (RRD), QPLD and IFD) and MM-GBSA calculations were followed to propose antagonists against nNOS with a wide variety of scoring functions that defines better binding affinities, orientations and free energies of the dock complexes and finally the best lead-nNOS complex and cocrystal-nNOS complex was subjected to $50 \mathrm{~ns}$ MD simulation studies, as it is the fundamental computational tool for capturing dynamic

*Corresponding author: Uma-Maheswari A, Associate Professor and Coordinator of BIF, SVIMS Bioinformatics Centre, Department of Bioinformatics, SVIMS University, Tirupati -517507, AP, India, Tel: 0877-2287727; E-mail: svims.btisnet@nic.in

Received: November 03, 2016; Accepted: January 21, 2017; Published January 24, 2017

Citation: Madhulitha NR, Pradeep N, Sandeep S, Hema K, Chiranjeevi P, et al. (2017) E-Pharmacophore Model Assisted Discovery of Novel Antagonists of nNOS. Biochem Anal Biochem 6: 307. doi: 10.4172/2161-1009.1000307

Copyright: $\odot 2016$ Madhulitha NR, et al. This is an open-access article distributed under the terms of the Creative Commons Attribution License, which permits unrestricted use, distribution, and reproduction in any medium, provided the original author and source are credited. 
events apart from the static structure-based approaches, such as docking and virtual screening, that have also made important strides in advancing drug discovery which would be useful for treating nNOS mediated Alzheimer's disease by designing novel and potent inhibitor.

\section{Materials and Methods}

Human nNOS possess 3 co-crystal structures $(4 \mathrm{D} 1 \mathrm{~N}, 4 \mathrm{UCH}$ and $4 \mathrm{~V} 3 \mathrm{U}$ ) with common $\mathrm{H} 4 \mathrm{~B}$ inhibitor in the protein data bank (PDB) (http://www.rcsb.org). Among the three structures, the lowest resolution $(4 \mathrm{D} 1 \mathrm{~N})$ structure was considered to propose antagonists through e-pharmacophore modeling, virtual screening, and multiple docking and molecular dynamics simulations.

\section{Active site analysis and protein structure preparation}

Active site was defined for further study as these residues contribute to the structural and functional properties of the protein. The active site residues of human nNOS were defined $4 \AA$ around co-crystallized inhibitor using PDBsum [11]. Protein structure was minimized using OPLS_2005 force field by converging the heavy atoms to RMSD of 0.3 $\AA$ A. Hydrogen atoms were added to all the atoms in the system and bond orders, formal charges were added for the hetero groups. Optimization was done at neutral pH. A $10 \times 10 \times 10 \AA$ grid was generated around the active site residues of $4 \mathrm{D} 1 \mathrm{~N}$ co-crystal structure and grid was used to generation of e-pharmacophore model and docking analysis.

\section{Generation of E-pharmacophore model}

Co-crystal ligand was docked in the generated grid using Glide XP docking. Glide XP descriptor information was retrieved from the refined ligand-receptor complex and the energy terms were mapped on to the atoms. Glide XP energies were added together for all the atoms which comprises the pharmacophoric sites. As fitness score is the measure of how well the ligand fits into the receptor with reference to the ligand. Pharmacophoric sites with fitness score less than -0.5 were rejected [12]. E-pharmacophore model of nNOS was generated from $4 \mathrm{D} 1 \mathrm{~N}$ docked complex using the protein ligand coordinates.

\section{Preparation of nNOS inhibitors library}

Generated e-pharmacophore model with the selected features was subjected to shape based similarity screening using PHASE v.3.9 (Phase, version 3.9, Schrödinger, LLC, New York, NY, 2014). Flexible search was performed in the in-house library against more than one million compounds from ChemBank, ChemPDB, KEGG ligand, Anti-HIV NCI, Drug-likeness NCI, Not annotated NCI, AKos GmbH and Asinex Ltd small molecule databases. The hits matched for the pharmacophoric features were considered with a distance matching tolerance of 3.0 A. Multiple conformers were generated and inactive compounds were rejected so that the false positives are reduced. All the hits obtained from the shape based similarity search based on e-pharmacophore model and $\mathrm{H} 4 \mathrm{~B}$ co-crystallized inhibitor were exported as library of nNOS inhibitors.

\section{Molecular docking}

In the present study, multiple docking protocols such as rigid receptor docking (RRD), quantum polarized ligand docking (QPLD) and induced fit docking (IFD) followed by binding free energy $(\Delta G)$ calculation by Prime/MM-GBSA analysis were performed to propose novel inhibitors for human nNOS. The ligands were prepared and chemical correctness was achieved to expand protonation, stereo chemical, ionization variations, energy minimization and tautomeric states at $\mathrm{pH} 7.0 \pm 2.0$ units using LigPrep [13] and Epik [14].
Ligands with reactive functional group and compounds with high ionization states or ligands that are not obeying Lipinski's rule of five were removed. The nNOS grid and the library of nNOS inhibitor compounds were imported into Maestro v.9.8 (Maestro, version 9.8, Schrödinger, LLC, New York, NY, 2014) and then docked into the active site of nNOS. Same protocol was followed for the co-crystal ligand used for generation the e-pharmacophore model so as to make a basis for comparison. RRD comprises multi-level docking steps such as high throughput virtual screening (HTVS), standard precision (SP) and extra precision (XP) docking to selectively filter the ligands at every stage from lower severity to higher severity of binding affinity [15]. Further, ligands obtained from XP docking were subjected to binding free energy $(\Delta \mathrm{G})$ calculation using Prime/MM-GBSA. Resulted ligands from XP docking and Prime/MM-GBSA analysis were subjected to QPLD docking by using Q-site module of Schrödinger, where quantum mechanical and molecular mechanical (QM/MM) calculations were calculated [16]. In QPLD initially, Glide SP docking was used to generate top five poses of each lead. Partial atomic charges were calculated for each selected poses of most energetically favorable bound leads towards nNOS. Then leads were redocked using Glide with the charge sets calculated earlier in Q-Site refinement and were subjected to binding free energy $(\Delta \mathrm{G})$ calculations. Subsequently, IFD protocol of Schrödinger were employed to the best docking complex obtained from QPLD and MM-GBSA analysis, by allowing flexibility to active site residues of nNOS. The Glide XP docking was used for the initial docking and 20 poses for lead 1 were retained. In the second step, Prime v.3.6 (Prime, version 3.6, Schrödinger, LLC, New York, NY, 2014) was used to generate the induced fit nNOS-lead 1 complex followed by the refinement of backbone and side chains of each docked conformations [17]. Prime energy was used to rank the nNOS-lead 1 refined complex. Finally in IFD, Glide XP was used to re-dock the lead 1 into the low energy conformation of nNOS refined earlier using Prime (Prime, version 3.6, Schrödinger, LLC, New York, NY, 2014). IFD scores were calculated by integrating nNOS-lead 1 interaction energy as well as the total energies of the system [18]. Binding free energy $(\Delta \mathrm{G})$ was calculated for the induced fit nNOS-lead 1 docked complex.

\section{Free energy calculation}

The docked complex from RRD, QPLD and IFD were subjected to binding free energy $(\Delta G)$ calculations for lead molecules with nNOS using Prime-MM/GBSA approach, as ranking of leads based on binding free energy would be effective than XP GScore. Using OPLS_2005 force field and generalized Born/surface area (GB/SA) continuum solvation model energies of the dock complexes were calculated. Instead of vdW surface, generalized Born/surface area model was employed for better representation of the solvent accessible surface area. The binding energy was calculated by the following equation.

\section{$\Delta \mathrm{Gbind}=\Delta \mathrm{E}+\Delta \mathrm{Gsolv}+\Delta \mathrm{GS}$}

Where, $\Delta \mathrm{E}$ is the sum of minimized energies of the receptor-ligand docking complex using the OPLS-2005. $\Delta \mathrm{G}_{\text {solv }}$ is the difference in the GBSA solvation energies of sum of the unaligned receptor and ligand with that of receptor-ligand docking complex. $\Delta \mathrm{G}_{\mathrm{SA}}$ is the difference in non-polar desolvation energy of the protein-ligand docking complex and the energies of the unaligned protein and ligand. $\Delta \mathrm{G}_{\mathrm{bind}}$ is the difference in the polar desolvation energy which is calculated by the generalized Born (GB) continuum model based on the defined within the $2 \AA$ regions around the ligand.

\section{Evaluation of virtual screening}

The virtual screening protocol [19] was validated by enrichment 


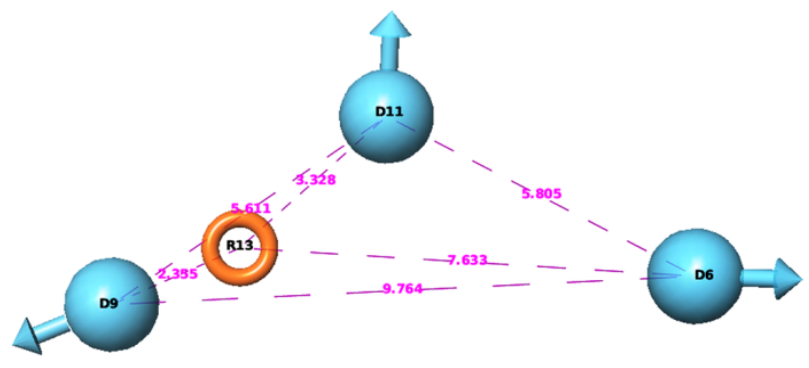

Figure 1: Four featured pharmacophore model.

\begin{tabular}{|c|c|c|c|c|c|c|c|c|c|}
\hline Lead & Mol. Wt. & Rotor & SASA & FOSA & WPSA & PISA & Volume & Donor & Acceptor \\
\hline Lead 1 & 344.21 & 4 & 540.26 & 87.05 & 70.62 & 259.41 & 923.15 & 3.5 & 4.5 \\
\hline Lead 2 & 330.18 & 4 & 520.53 & 0 & 77.28 & 292.31 & 871.45 & 4 & 4.5 \\
\hline Lead 3 & 300.33 & 5 & 525.84 & 0 & 54.02 & 320.37 & 894.61 & 4 & 4.25 \\
\hline Co-crystal ligand H4B & 237.22 & 2 & 455.256 & 73.119 & 0 & 94.236 & 731.385 & 3 & 3 \\
\hline \multicolumn{5}{|c|}{ Parameters } & \multicolumn{5}{|c|}{ (Range $95 \%$ of Drugs) } \\
\hline \multicolumn{5}{|c|}{ Mol. Wt. = Molecular Weight } & \multicolumn{5}{|c|}{$(130.0 / 725.0)$} \\
\hline \multicolumn{5}{|c|}{ Rotor $=$ No. of Rotatable Bonds } & \multicolumn{5}{|c|}{$(0.0 / 15.0)$} \\
\hline \multicolumn{5}{|c|}{ SASA = Total solvent accessible surface area } & \multicolumn{5}{|c|}{$(300.0 / 1000.0)$} \\
\hline \multicolumn{5}{|c|}{ FOSA = Hydrophobic solvent accessible surface area } & \multicolumn{5}{|c|}{$(0.0 / 750.0)$} \\
\hline \multicolumn{5}{|c|}{ PISA = Carbon Pi solvent accessible surface area } & \multicolumn{5}{|c|}{$(0.0 / 450.0)$} \\
\hline \multicolumn{5}{|c|}{ WPSA= Weakly Polar solvent accessible surface area } & \multicolumn{5}{|c|}{$(0.0 / 175.0)$} \\
\hline \multicolumn{5}{|c|}{ Volume = Molecular Volume $\left(\mathrm{A}^{\wedge} 3\right)$} & \multicolumn{5}{|c|}{$(500.0 / 2000.0)$} \\
\hline \multicolumn{5}{|c|}{ Donor = Donor - Hydrogen Bonds } & \multicolumn{5}{|c|}{$(0.0 / 6.0)$} \\
\hline \multicolumn{5}{|c|}{ Acceptor = Acceptor - Hydrogen Bonds } & \multicolumn{5}{|c|}{$(2.0 / 20.0)$} \\
\hline
\end{tabular}

The abbreviations used to specify different principal descriptors of three probable leads to Table 2 and their range in $95 \%$ of the available drugs are given. The range for properties of 95\% drug is given based on QikProp.

Table 1: Principle descriptors of three proposed leads and co-crystal ligand towards human nNOS.

factor (EF) metrics calculations. Co-crystal ligand and obtained 3 leads were considered as actives which were combined to decoys set of 1000 compounds downloaded from Schrodinger to form an internal library of 1004 compounds. The ability of the e-pharmacophore to distinguish the active site compounds from decoys was evaluated for validating the virtual screening protocol. Availed results were interpreted with enrichment factor at $1 \%$ that shows the enhanced recovery of known actives over the decoys. Boltzmann-enhanced discrimination of receiver operating characteristic curve (ROC) i.e., BEDROC $(\alpha=20.0)$ [20] metrics which shows the enhanced recognition of known actives over the decoys from the internal database.

\section{Molecular dynamics simulations}

Molecular dynamics (MD) simulations is a fully flexible method that offers wide range of solutions such as to refine protein-ligand complexes, evaluating stability of docking complexes and calculating the binding energies during the simulations run [20,21]. Desmond v.3.8 of Schrodinger was used to compute the energies and intermolecular interactions. nNOS-lead 1 docked complex system was embedded in the SPC water model and assigned with OPLS-AA 2005 force field. System was neutralized with the counter ions and specified with periodic boundary conditions such as particle mesh Ewald (PME) method for electrostatics, Lennard-Jones interactions limiting the cutoff to $10 \AA$ and SHAKE algorithm for limiting the movements all hydrogen atoms involved in the covalent bonds. The neutralized system was energy minimized with steepest descent method with a maximum of 2000 steps with solute restrains followed by another 2000 steps without solute restraints. Then 12 ps simulation was performed in NVT ensemble followed by NPT ensemble for restraining nonhydrogen solute atoms at $10 \mathrm{~K}$ temperature. Further, NPT ensemble was carried out for $24 \mathrm{ps}$ simulation at $300 \mathrm{~K}$ temperature with solute non-hydrogen atoms subsequently for the solute non-hydrogen atoms with no restraints. During the initial simulations, Berendsen thermostats and barostat were applied to control the temperatures and pressures. The relaxed system was subjected to $50 \mathrm{~ns}$ simulations and trajectory was recorded with a time interval of $4.8 \mathrm{ps}$. Intermolecular hydrogen bond interactions, energy potential and root mean square deviation (RMSD) were examined to define the stability of nNOS-lead 1docked complex.

\section{Results and Discussion}

\section{Receptor preparation and active site analysis}

Active site residues were defined around $4 \AA$ region of the co-crystal ligand of $4 \mathrm{D} 1 \mathrm{~N}$. Further the active site residues of receptor were cross checked with PDBsum. Residues such as Arg601, Val682, Trp683, Val685 and Hem750, constitutes active site of nNOS and were present deep into the binding cleft between $\mathrm{N}$-terminal and C-terminal lobes.

\section{E-pharmacophore generation}

As inhibitor co-crystal structures is available for nNOSin the PDB, energy-optimized structure based pharmacophore method was practiced in the present study. Pharmacophoric site was based on the structural and interactional energy information between the nNOS and the co-crystal ligand. E-pharmacophore was written with the selected features such that, it could effectively map all the structural requirements that are pharmacophoric features which were responsible for nNOS bioactivity. E-pharmacophore was developed from nNOS docked complex such that all the Glide XP energetic terms were mapped. The derived e-pharmacophore model has four features 
a
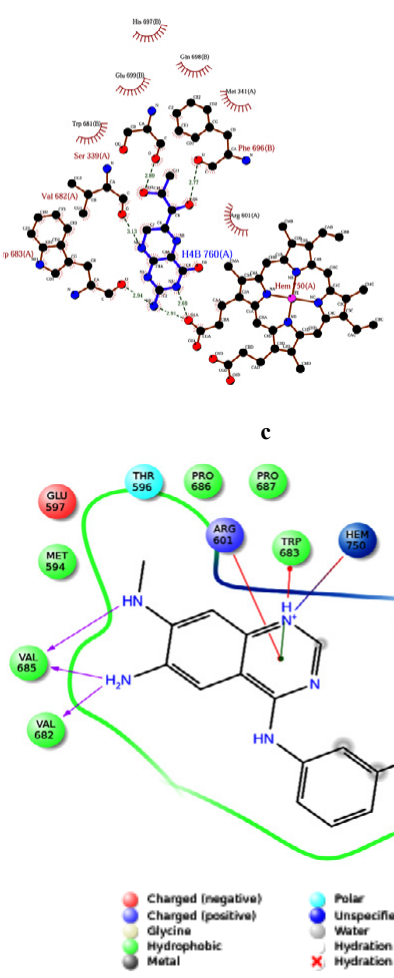

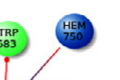

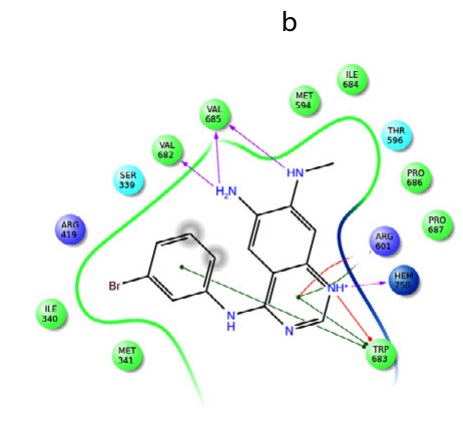

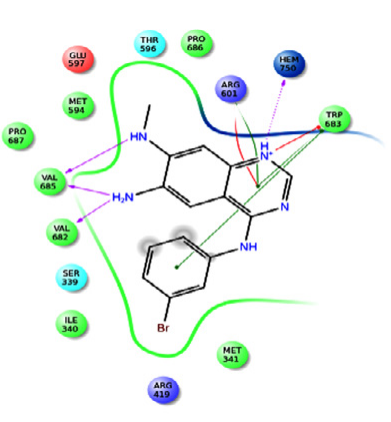

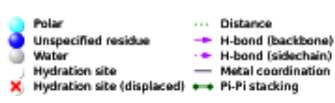

- Salt bribse
Solwent exposicre

Figure 2: Interaction patterns of nNOS with (a) Co-crystal ligand (b) Lead 1-nNOS docked complex in RRD (c) Lead 1 nNOS docked complex in QPLD (d) Lead 1-nNOS docked complex in IFD.

(RDDD) such as one aromatic ring (R) and three hydrogen bond donors (D). The pharmacophoric sites having fitness score more than -0.5 were written as pharmacophore hypothesis as it measures how well the pharmacophore site points are aligned with those of the co-crystal ligand. The e-pharmacophore model and their pharmacophoric sites were shown in (Figure 1).

\section{Ligand optimization}

The generated e-pharmacophore model was used for shape based similarity screening against the in-house library of small molecules. Shape screening based on e-pharmacophore results 2701 structurally similar compounds. Co-crystal inhibitor along with the shape screened compounds was exported as library of nNOS inhibitors. 2301 compounds have passed the Lipinski's filter, subsequently subjected to reactive filters resulted 2201 compounds which were considered for docking analysis and binding energy calculations.

\section{Docking}

The RRD docking was streamlined through HTVS, SP and XP docking methods to find potential ligand molecules using prepared grid and 2201 compounds library of nNOS inhibitors. 2201 compounds were docked in HTVS resulted 220 hits and further passed to SP docking. These resulted 22 hits from SP were redocked to nNOS using XP docking and Prime/MM-GBSA was performed. The 22 ligands were subjected to quantum polarized ligand docking (QPLD), for evaluating the strength of relative active site interactions of each potential ligands with nNOS by accurate charge calculations followed by Prime/MMGBSA. Leads obtained from QPLD MM-GBSA were compared with the co-crystal structure.
The different docking strategies of RRD and QPLD followed by re-scoring with MM-GBSA affirmed the ability of three leads as a potent nNOS inhibitor. QPLD docking and MM-GBSA analysis results showed that, 3 leads having the good binding free energy interactions and having the better Glide scores. Three leads having hydrogen bond interactions with nNOS, lead 1 possessed highest binding affinity towards nNOS with QPLD $\Delta \mathrm{G}$ score of $-61.931 \mathrm{kcal} / \mathrm{mol}$; Glide Score of $-10.45 \mathrm{kcal} / \mathrm{mol}$ when compared to co-crystal ligand (Table 1). Induced fit docking (IFD) protocol of Schrödinger was considered to calculate the possible binding modes and associated conformational changes within the receptor active site by using Glide and Prime. The lead 1 was observed to exhibit highest binding affinity and binding free energy towards nNOS that are calculated using MM-GBSA (Glide Score as $-11.53 \mathrm{kcal} / \mathrm{mol} ; \Delta G$ value as $-61.93 \mathrm{kcal} / \mathrm{mol}$ ) (Table 1$)$. Lead 1 interaction in IFD docking mode with nNOS (Figure 2c).

\section{Interactions of nNOS-lead 1 docking complex}

Multiple docking strategies revealed that lead 1 is having the binding free energy and docking score than the co-crystal ligand and obtained three leads (Table 1) (Figures $2 \mathrm{~b}, 2 \mathrm{c}$ and $2 \mathrm{~d}$ ). The good binding affinity of lead 1 is due to hydrogen bonding, hydrophobic interactions, hydrophilic interactions, electrostatic interactions and steric interactions with Glide Score of $-8.04 \mathrm{kcal} / \mathrm{mol} ; \Delta G$ value as $-61.93 \mathrm{kcal} / \mathrm{mol}$ in RRD. Lead 1 bound to the nNOS with four hydrogen bond interactions, in which two hydrogen bonds were observed with backbone residue of Val685 and hydrogen bond was formed with the side chain residue of Hem750 and another with backbone residues of Val682. Lead 1 also involved in two $\pi-\pi$ stacking interactions and two $\pi$-cation interactions with Arg601 and Trp683. Lead 1 docking interactions were well collaborated with co-crystal structure of $4 \mathrm{D} 1 \mathrm{~N}$ 


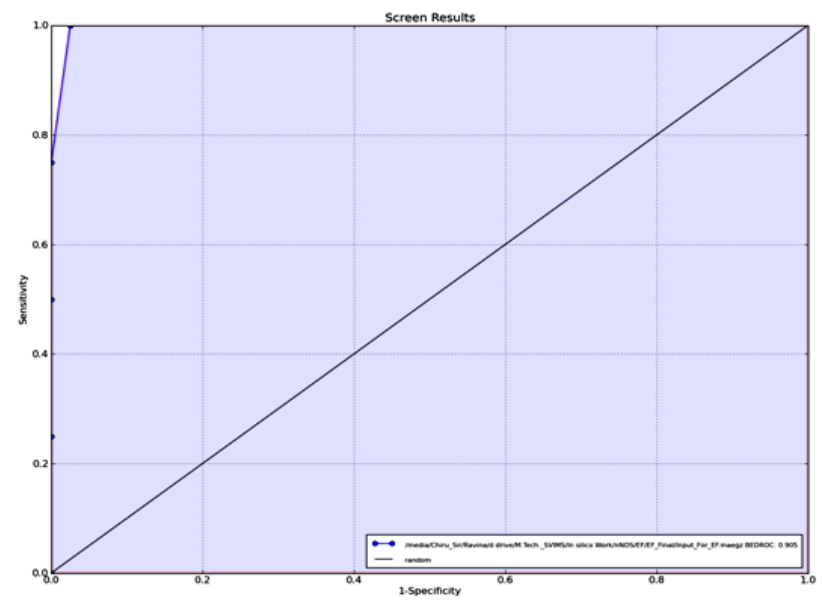

Figure 3: Enrichment curve for evaluating virtual screening protocol.
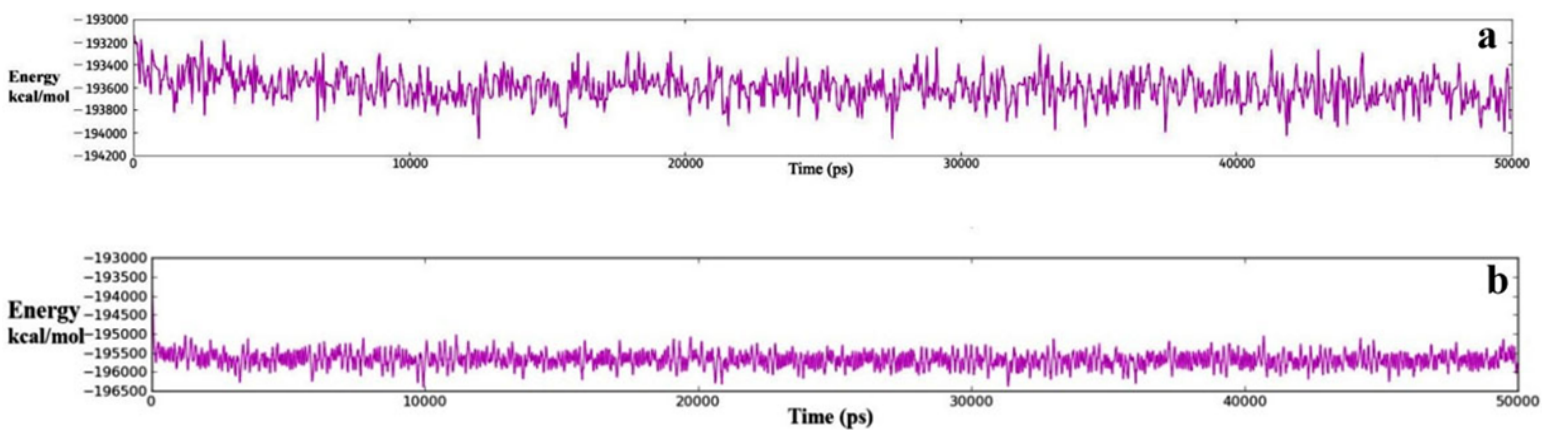

Figure 4: Potential energy plot of (a) nNOS-H4B (b) nNOS-lead 1 complex during 50 ns MD simulations run.

(Figures $2 \mathrm{a}$ and $2 \mathrm{~b}$ ). Molecular interactions of nNOS-lead 1 docking complex showed four hydrogen bonds, two $\pi-\pi$ stacking and two $\pi$-cation interactions revealing high stability (Figure $2 b$ ). Active site interactions observed in RRD were reproduced after QPLD and MMGBSA analysis where lead 1 showed the Glide Score of $-10.45 \mathrm{kcal} / \mathrm{mol}$; $\Delta G$ value as $-61.93 \mathrm{kcal} / \mathrm{mol}$ in QPLD. Lead 1 showed two hydrogen bonds were observed with backbone residue of Val685 and single hydrogen was formed with side chain residue of Hem750 and another with backbone residue of Val682. Two $\pi-\pi$ stacking interactions and single $\pi$-cation interaction were observed with Trp683 and single $\pi-\pi$ stacking, $\pi$-cation interaction was observed with Arg601 (Figure 2c). Active site interactions observed in QPLD were reproduced after IFD and MM-GBSA analysis (Figure 2d) and lead 1 showed the Glide Score of $-11.53 \mathrm{kcal} / \mathrm{mol} ; \Delta G$ value as $-61.93 \mathrm{kcal} / \mathrm{mol}$ in QPLD. Docked complex showed single hydrogen bond with Val682 and Val 685, one $\pi-\pi$ stacking, $\pi$-cation with Trp683, one $\pi$-cation interaction with Arg601 and salt bridge was formed with Hem750.

\section{Evaluation of virtual screening}

One co-crystal ligand and three leads (actives) were screened against internal library of 1000 decoys and actives resulted from XP docking with nNOS, yielded $75 \%$ of known actives which were within EF1\% of internal library containing actives and decoys (EF 1\%=75). Receiver operative characteristic curve (ROC) metric corresponds to a linearly scaled average of the actives positions with ordered ranks among the internal library. The range of ROC was 0 to 1 and Truchon and Bayly considered ROC with $\geq 0.7$ as a desirable performance value.
EF metrics measures the early recognition of actives found within the defined ordered list relative to the randomly distributed decoys in the internal library. In virtual screening, all the actives were found with ROC of 0.99 with respective to nNOS e-pharmacophore model. BEDROC value of 0.905 constitutes the scope of early identification of actives from the ranked compounds in the internal library. Resulted $\mathrm{EF}, \mathrm{ROC}$ and BEDROC values were 75 (EF1\%), 0.99, $0.905(\alpha=20.0)$ respectively indicates that the generated e-pharmacophore model was considered for virtual screening against in-house library were enough in retrieving the active compounds. The enrichment curve graphically represents quality of retrieved actives which were ranked after comparing to decoys within internal library (Figure 3).

\section{Molecular dynamics simulations}

MD simulations were performed to know inter-atomic interactions that facilitate the complex stability. MD simulations studies were performed up to $50 \mathrm{~ns}$ to analyze the conformational stability and steady nature of nNOS-lead 1 docked complex and nNOS-H4B docked complex in solvated model system. After simulations, energy plot reveals that the nNOS-lead 1 docking complex was comparatively consistent during $50 \mathrm{~ns}$ MD simulations run (Figure 4a). Stability of the docked complex was compared with the $4 \mathrm{D} 1 \mathrm{~N}-\mathrm{H} 4 \mathrm{Bcomplex}$ throughout the 50 ns simulations run.

The energies of both the systems were relatively stable during 50 ns simulations run. Energy of both the simulated docked complexes showed that nNOS-lead 1 complex was having the lowest energy when compared with nNOS-H4B complex and energy of the system was 
Citation: Madhulitha NR, Pradeep N, Sandeep S, Hema K, Chiranjeevi P, et al. (2017) E-Pharmacophore Model Assisted Discovery of Novel Antagonists of nNOS. Biochem Anal Biochem 6: 307. doi: 10.4172/2161-1009.1000307

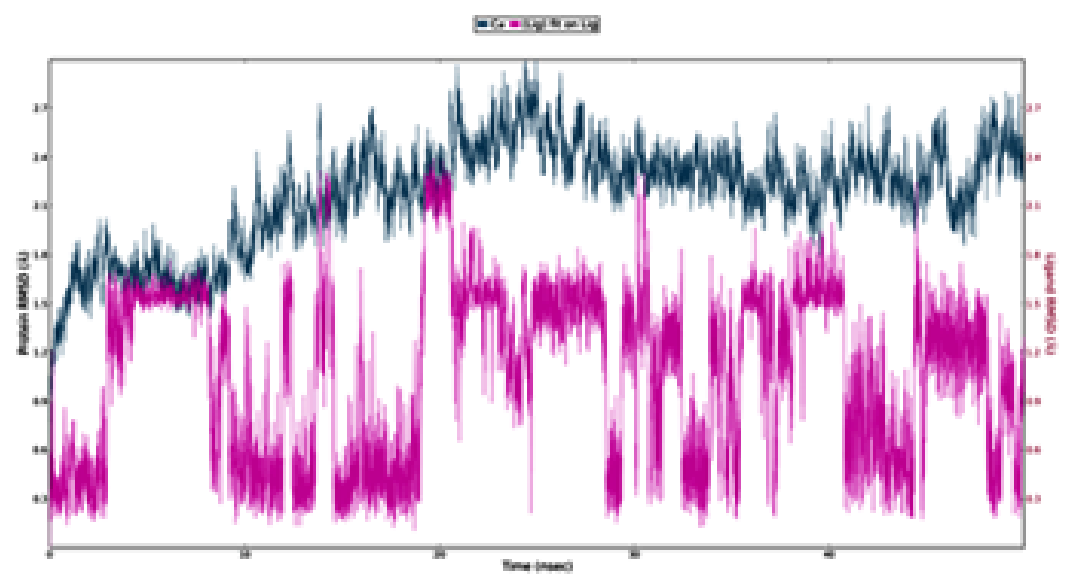

b

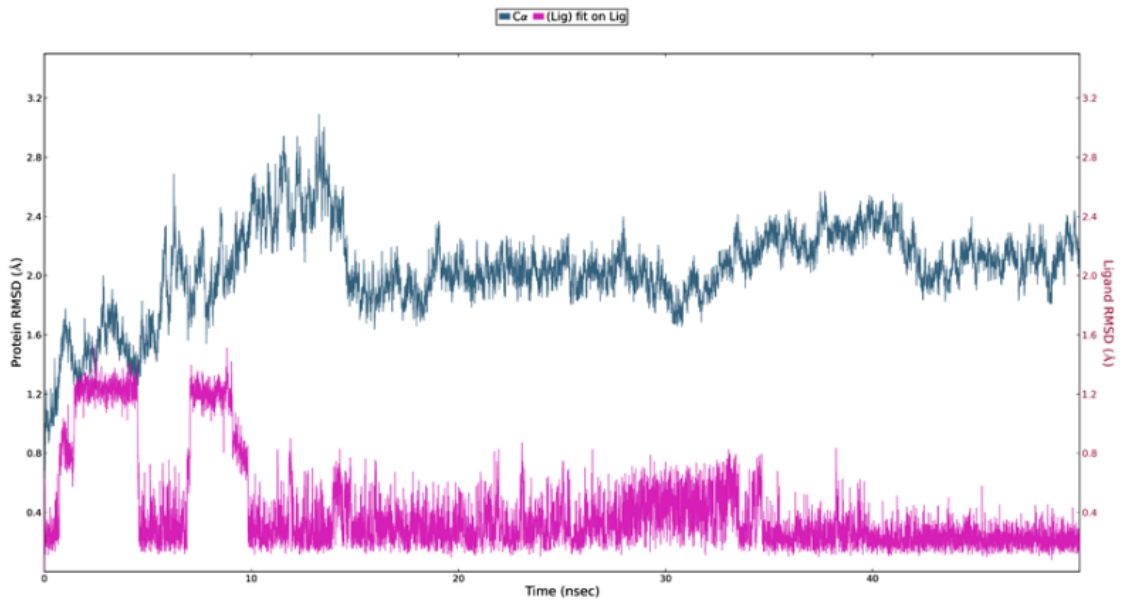

Figure 5: RMSD plot of (a) H4B-nNOS (b) lead 1-nNOSduring 50 ns MD simulations run.

relatively consistent during $50 \mathrm{~ns} \mathrm{MD}$ simulations (Figure $4 \mathrm{~b}$ ). The root mean square deviation range for $\mathrm{nNOS} \mathrm{C} \alpha$ and lead 1 complex initial conformation and during simulations period were ranged from 0.70 $\AA$ to $2.99 \AA$ for $\mathrm{C} a$ of nNOS and for lead 1 it was ranged from $0.10 \AA$ to $2.39 \AA$, the average RMSD of Ca was $2.12 \AA$ and for lead 1 is 1.06 $\AA$ in 50 ns simulations (Figure $5 b$ ). The analysis of the RMSD plot for $\mathrm{C} \alpha$ and heavy atoms of lead 1 showed that after a small rearrangement from the initial conformation of complex was stable during $50 \mathrm{~ns} \mathrm{MD}$ simulations period. In case of nNOS-H4B docked complex, RMSD range for nNOS backbone and $\mathrm{H} 4 \mathrm{~B}$ were within conventional range from $0.08 \AA$ to $1.53 \AA$ and $0.67 \AA$ to $3.09 \AA$ respectively, which is stable throughout MD simulations run. Average RMSD for nNOS backbone was $2.04 \AA$ and an average RMSD for lead 1 was $0.41 \AA$ (Figure 5a).

Root mean square fluctuations (RMSF) were useful for characterizing local changes along the protein chain and average RMSF for nNOS backbone and side chain residues at were within the limit of $1.13 \AA$ and $1.62 \AA$ respectively. RMSF for active site residues of nNOS were within the limit of $4.0 \AA$. Lower the RMSF values indicate the minimal fluctuations of backbone and side chain residues during the MD simulations run (Figure 6a).

In case of nNOS-H4B docked complex, RMSF of backbone and side chain residues at active site were within the limit of $1.01 \AA$ and $1.50 \AA$ respectively. Therefore, the lower RMSD and RMSF values of the nNOS-lead 1 system shows the smaller structural rearrangements and lesser internal motions around the active site residues of nNOS and it was better collaborated with the nNOS-H4B docked complex (Figure 6b).

\section{Binding interactions of nNOS-lead 1 and nNOS-H4B com- plexes during $50 \mathrm{~ns}$ simulations}

nNOS-lead 1 and nNOS-H4B docking interactions were analyzed throughout 50 ns run period (10416 trajectories). During the simulations, lead 1 formed hydrogen bond (H-bond) interactions with the active site residues Trp683, Val685, three $\pi-\pi$ stacking with Trp683, one $\pi-\pi$ stacking with Arg601 and two $\pi$-cation interactions with Arg601in 10416 trajectories with nNOS protein, which revealed the stability of nNOS-lead 1 complex throughout the 50 ns simulations run (Figure 7b). Total 10416 trajectories were analyzed during the $50 \mathrm{~ns}$ simulations to check all types of binding interactions of lead 1 with nNOS. Lead 1 showed hydrogen bonding interactions in 2083 trajectories, water mediated interactions in 850 trajectories and 5800 trajectories involved in hydrophobic contacts with the Trp683 of nNOS (Figure 7b). In case of Val 685, lead 1 showed hydrogen bonding interactions in 3800 trajectories, water mediated interactions in 1000 trajectories and 3800 trajectories involved in hydrophobic contacts 

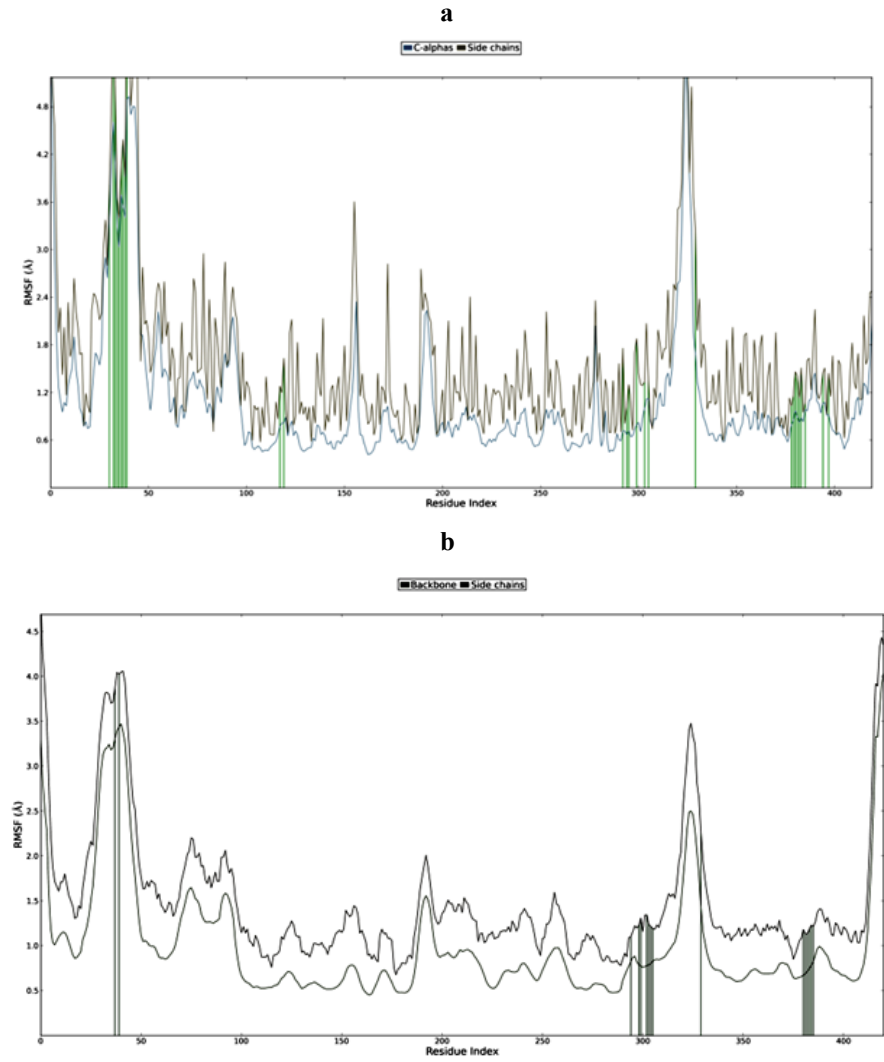

Figure 6: RMSF plot (a) nNOS-H4B (b) nNOS-lead 1.

a

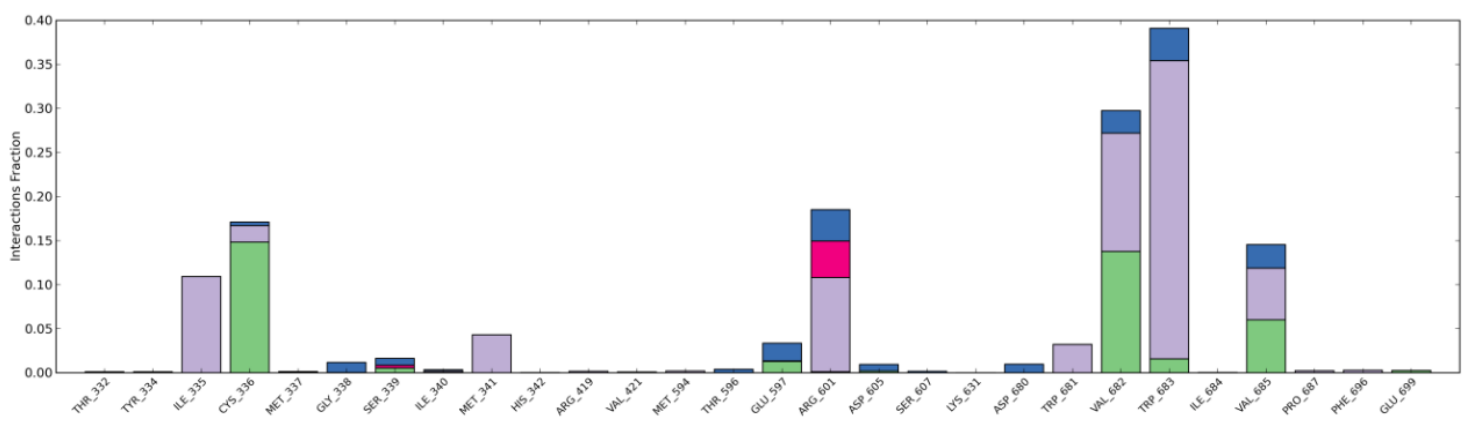

b

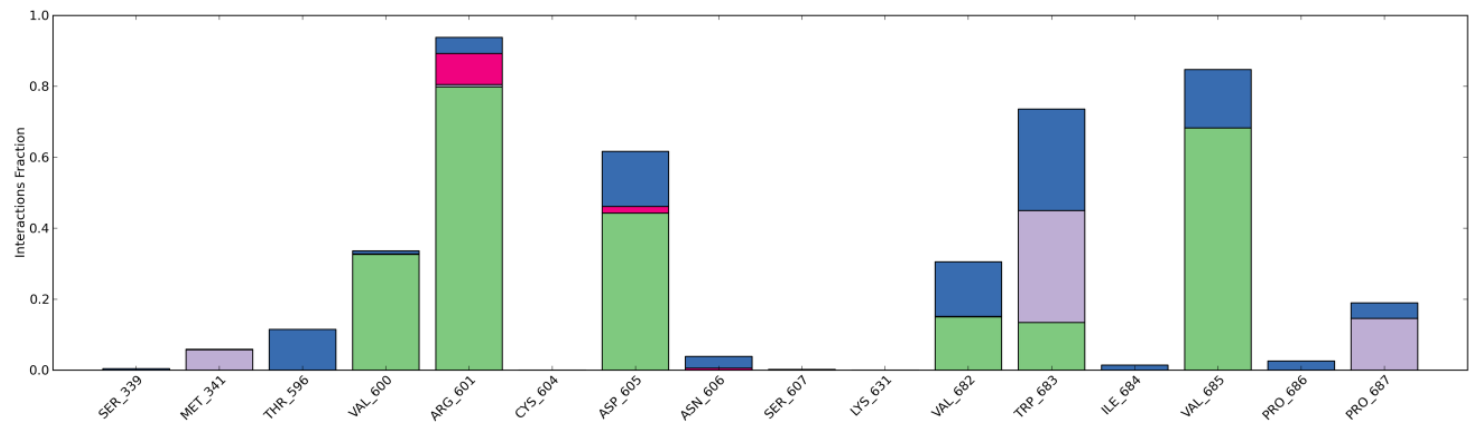

Figure 7: Interaction patterns after $50 \mathrm{~ns}$ MD simulations run(a)nNOS-H4B (b)nNOS-lead 1 complex (Yaxis $0.2 \%=2083$ trajectories). 
Citation: Madhulitha NR, Pradeep N, Sandeep S, Hema K, Chiranjeevi P, et al. (2017) E-Pharmacophore Model Assisted Discovery of Novel Antagonists of nNOS. Biochem Anal Biochem 6: 307. doi: 10.4172/2161-1009.1000307

Page 8 of 9

\begin{tabular}{|c|c|c|c|c|c|c|c|c|}
\hline Lead & Log Po/w & $\log S$ & Clog S & $\log B B$ & Rule of 5 & Rule of 3 & $\log \mathrm{Kp}$ & Log Khsa \\
\hline Lead1 & 2.44 & -3.908 & -5.133 & -0.7 & 0 & 0 & -2.623 & -0.047 \\
\hline Lead2 & 2.44 & -3.908 & -5.133 & -0.7 & 0 & 0 & -2.623 & -0.047 \\
\hline Lead3 & 2.112 & -3.387 & -4.369 & -0.99 & 0 & 0 & -2.702 & -0.172 \\
\hline Co-crystal ligand H4B & -1.19 & -0.643 & -1.409 & -1.926 & 0 & 1 & -1.129 & -0.688 \\
\hline \multicolumn{5}{|c|}{ Parameters } & \multicolumn{4}{|c|}{ (Range $95 \%$ of Drugs) } \\
\hline \multicolumn{5}{|c|}{$\log \mathrm{P}$ o/w $=\log \mathrm{P}$ for octavo/water } & \multicolumn{4}{|c|}{$(-2.0 / 6.5)$} \\
\hline \multicolumn{5}{|c|}{ Logs $=\log S$ for aqueous solubility } & \multicolumn{4}{|c|}{$(-6.5 / 0.5)$} \\
\hline \multicolumn{5}{|c|}{$\mathrm{Cl} \log \mathrm{S}=\log \mathrm{S}-$ conformation independent } & \multicolumn{4}{|c|}{$(-6.5 / 0.5)$} \\
\hline \multicolumn{5}{|c|}{$\log B B=\log B B$ for brain $/$ blood } & \multicolumn{4}{|c|}{$(-3.0 / 1.2)$} \\
\hline \multicolumn{5}{|c|}{ Log KP $=\log K P$ for skin permeability } & \multicolumn{4}{|c|}{ (KP in $\mathrm{cm} / \mathrm{hr}$ ) } \\
\hline \multicolumn{5}{|c|}{ Log $\mathrm{K}$ hsa $=\log \mathrm{K}$ hsa Serum protein binding } & \multicolumn{4}{|c|}{$(-1.5 / 1.5)$} \\
\hline \multicolumn{5}{|c|}{ Lipinski rule of 5 violations } & \multicolumn{4}{|c|}{ (maximum is 4 ) } \\
\hline \multicolumn{5}{|c|}{ Jorgensen rule of 3 violations } & \multicolumn{4}{|c|}{ (maximum is 3 ) } \\
\hline
\end{tabular}

Table 2: Predicted ADME properties of three leads and co-crystal ligand towards human nNOS.
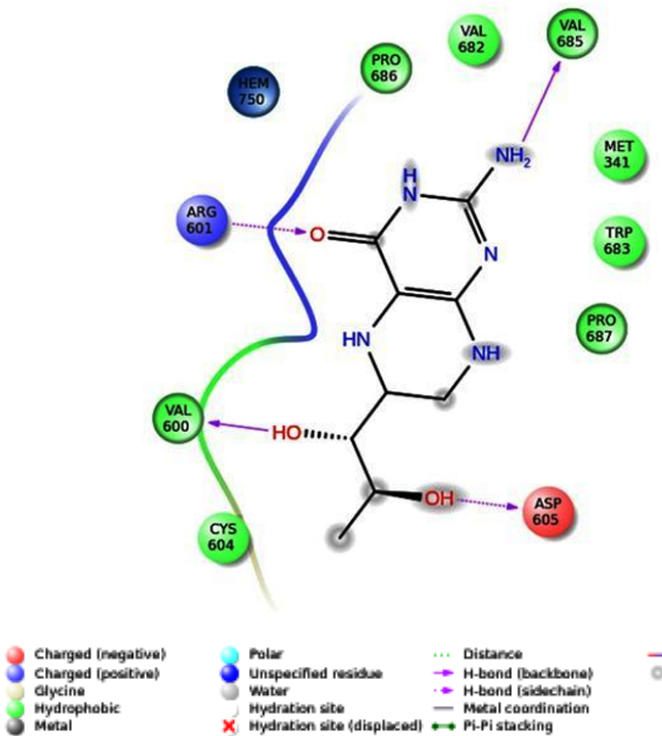

(isplized) $\rightarrow$ Pi.Pistacking

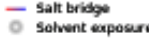

Figure 8: Analysis of (a) nNOS-H4B (b) nNOS-lead 1 contacts towards nNOS during 50 ns simulations run.

(Figure 7b). Whereas, lead 1 involved in hydrogen bonding interactions in 3800 trajectories, hydrophobic contacts in 3800 trajectories and 400 trajectories involved in water mediated interactions with the Val682 (Figure 7b). In case of Arg601, lead 1 involved in hydrogen bonding interactions in 3750 trajectories, ionic contacts in 1200 trajectories and 1000 trajectories involved in hydrophobic interactions (Figure 7b). Average analysis revealed that out of 10416 trajectories lead 1 is having better interactions with Val685 in 8600 trajectories, in 8000 trajectories bonding with the Val682, in 8733 trajectories with the Trp683 and Arg601 involved bonding in 5900 trajectories (Figure 7b).

The nNOS-H4B complex displayed four hydrogen bonds with active site residues such as Val600, Arg601, Asp605 and Val685 during MD simulations run (Figure 7a) 10416 trajectories were analyzed during the $50 \mathrm{~ns}$ simulations and $\mathrm{H} 4 \mathrm{~B}$ showed hydrogen bonding interactions in 3620 trajectories with the Val600 (Figure 7a). Whereas with Arg605, $\mathrm{H} 4 \mathrm{~B}$ involved hydrogen bonding interactions in 4320 trajectories, water mediated interactions in 1520 trajectories and 100 trajectories involved in ionic contacts (Figure 7a) H4B formed hydrogen bonding interactions in 8000 trajectories, ionic contacts in 1000 trajectories and 250 trajectories involved in water mediated interactions with the Arg601 (Figure 7a).

In case of Val685, H4B involved in hydrogen bonding interactions in 6200 trajectories and 1500 trajectories were involved in water mediated interactions (Figure 7a). Compare to the crystal ligand data lead 1 data revealed strongly binding with the active site residues of nNOS in over all $50 \mathrm{~ns}$ simulations run and nNOS-lead 1 complex well collaborated with the binding free energy in different docking strategies such as RRD, QPLD and IFD and also in $50 \mathrm{~ns}$ MD simulations run.

The analysis of RMSD and RMSF confirmed the lesser rearrangement in the protein backbone and stable potential energy of docking complex revealed that nNOS-lead 1 complex was more stable in the solvated model conditions throughout the 10416 trajectories. The proposed three leads showed favorable ADME/T properties against nNOS (Tables 1 and 2). 
Lead 1 formed better docking score and good active site interactions with nNOS than with the co-crystal docking complex by forming hydrogen bonds with active site residues such as Trp683, Val685, three $\pi-\pi$ stacking with Trp683, one $\pi-\pi$ stacking with Arg601 and two $\pi$-cation interactions with Arg601 of nNOS (Figure 8a). Stability of protein-ligand docked complex was analyzed in MD simulations through solvating water molecules because it was recognized closer to the physiological environmental conditions. Binding interactions of the best ranked docked complex were also observed during the 50 ns MD simulations run and analyzed for stability of docked complex in every trajectory. The nNOS-lead 1 docked complex was consistent throughout $50 \mathrm{~ns}$ simulations period. RMSD, RMSF and potential energy of docked complex were also in the acceptable range. In the present study, lead 1 showed molecular interactions with residues Arg601, Val-682, Trp-683 and Val-685 when compared to the co-crystal ligand and it was instable orientation with better binding affinity in RRD, QPLD, IFD and MD simulations.

Lead 1 showed favorable interactions with the active site residues along with the residues extended by hydrophobic interactions and water bridges, which results the conformational change within the active site of nNOS (Figure 8b). Therefore, blocking the active site with lead 1 was adequate to decrease the biological activity of nNOS with the unavailability of active site residues. Thus, nNOS induced neurofibrillary tangles formation subsequently neuronal apoptosis within the neurons of front-temporal region of brain might be reduced.

\section{Conclusion}

Altered expression of nNOS results in hyper phosphorylation of cytoplasmic protein tau in turn results in destabilization of microtubule ensuring the formation of Neurofibrillary tangles (NFTs). Thus, these NFTs forms a physical road blocks in the neurons and leads to neuronal apoptosis. As three crystal structures available for nNOS, e-pharmacophore modeling and multiple docking methods were practiced to define the leads. The obtained leads from multiple docking studies were analyzed for ADME/T properties to propose the leads. E-pharmacophore model of nNOS-ligand complex demonstrates the structural requirements of the nNOS to define potent leads. The analysis of nNOS-ligands complexes revealed the key amino acids present in the active site of nNOS that are important for ligand binding.

The generated e-pharmacophore was used for shape based similarity screening against in-house library and multiple docking was performed with the generated library of nNOS inhibitors. Results from docking strategies such as RRD, QPLD and IFD followed by MM-GBSA calculations revealed three the best leads when compared to the existing antagonists. MD simulations studies revealed the stability of lead 1 to form nNOS-lead 1 complex, such that it may reduce the nNOS biological activity by blocking the critical active site residues. The proposed three leads exhibited similar interaction pattern with increased binding affinity and favourable orientation towards nNOS, such that critical active sites were blocked in turn to reduce the biological activity of nNOS. The proposed three leads were also validated and ranked better than the existing co-crystal ligand and decoys in ROC metrics. Thus inhibition of nNOS results in reduced NFT formation in turn reduces neuronal loss in the fronto-temporal region of brain and pave a way for treating nNOS mediated AD.

\section{Acknowledgments}

Authors are thankful to DBT, Ministry of Science and Technology, Government of India, New Delhi for supporting the work through BTISnetBIF program (No. BT/ $\mathrm{BI} / 25 / 001 / 2006)$.

\section{References}

1. Waldemar G, Dubois B, Emre M, Georges J, McKeith IG, et al. (2007) Recommendations for the diagnosis and management of Alzheimer's disease and other disorders associated with dementia: EFNS Guideline. J Neuroophthalmol 14: 1-26

2. Jiang ZG, Ghanbari HA (2013) Oxidative stress and neurodegenerative disorders. Int J Mol Sci. 14: 24438-24475.

3. Serrano-Pozo A, Frosch MP, Masliah E, Hyman BT (2011) Neuropathological alterations in Alzheimer's disease. Cold Spring HarbPerspect Med 1: 006189.

4. Lace G, Savva GM, Forster G, De Silva R, Brayne C, et al. (2009) Hippocampa tau pathology is related to neuroanatomical connections: an ageing populationbased study. Brain. 132: 1324-1334.

5. Massaad CA, Klann E (2011) Reactive oxygen species in the regulation of synaptic plasticity and memory. Anticancer Res. 14: 1682-1897.

6. Hung YH, Bush Al, LaFontaine S (2013) Links between copper and cholesterol in Alzheimer's disease. FrontPhysiol: 4: 111.

7. Yang DS, Kumar A, Stavrides P, Peterson J, Peterhoff CM, et al. (2008) Neuronal apoptosis and autophagy cross talk in aging PS/APP mice, a model of Alzheimer's disease. Am J Pathol 173: 665-681.

8. Salcedo-Tello P, Ortiz-Matamoros A, Arias C (2011) GSK3 function in the brain during development, neuronal plasticity, and neurodegeneration. Int $J$ Alzheimers Dis 189-728.

9. Gorman AM (2008) Neuronal cell death in neurodegenerative diseases: Recurring themes around protein handling. J Cell Mol Med 12: 2263-2280.

10. Bobba A, Petragallo VA, Marra E, Atlante A (2010) Alzheimer's proteins, oxidative stress, and mitochondrial dysfunction interplay in a neuronal model of Alzheimer's disease. Int J Alzheimers Dis. 621-870.

11. Hooper C, Killick R, Lovestone S (2008) The GSK3 hypothesis of Alzheimer's disease. J Neurochem 104: 1433-1439.

12. Laskowski RA (2001) PDB sum: Summaries and analyses of PDB structures. Nucleic Acids Res 29: 221-222.

13. Pradeep N, Munikumar M, Swargam S, Hema K, Sudheer Kumar K, et al. (2015) 197 Combinations of e-pharmacophore modeling, multiple docking strategies and molecular dynamic simulations to discover of novel antagonists of BACE1. J Biomol Struct Dyn 1: 129-133.

14. Friesner RA, Banks JL, Murphy RB (2004) Glide: A new approach for rapid accurate docking and scoring: Method and assessment of docking accuracy. J Med Chem 47: 1739-1749.

15. Brooks WH, Daniel KG, Sung SS, Guida WC (2008) Computational validation of the importance of absolute stereochemistry in virtual screening. J Chem Inf Model 48: 639-645.

16. Shelley JC, Cholleti A, Frye LL (2007) Epik: A software program for pK(a) prediction and protonation state generation for drug-like molecules. J Comput Aided Mol Des 21: 681-691.

17. Das D, Koh Y, Tojo Y (2009) Prediction of potency of protease inhibitors using free energy simulations with polarizable quantum mechanics-based ligand charges and a hybrid water model. J Chem Inf Model 49: 2851-2862.

18. Mobley DL, Dill KA (2009) Binding of small-molecule ligands to proteins: "what you see; is not always; what you get." Structure 17: 489-498.

19. Cho AE, Guallar V, Berne BJ, Friesner R (2005) Importance of accurate charges in molecular docking: quantum mechanical/molecular mechanical (QM/MM) approach. J Comput Chem 26: 915-931.

20. Sudheer Kumar K, Pradeep N, Sandeep S, Hema K, Chiranjeevi P, et al. (2016) Inhibitor design against JNK1 through e-pharmacophore modeling docking and molecular dynamics simulations. J Recept Signal Transduct Res 36: 558-571.

21. Chiranjeevi P, Sandeep S, Pradeep N, Hema K, Sudheer Kumar K, et al. (2016) Inhibitor design for VacA toxin of Helicobacter pylori. J Proteomics Bioinform 9: 220-225. 